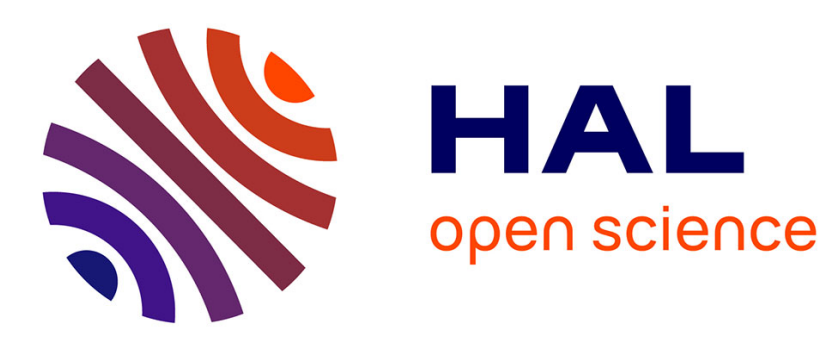

\title{
Robust inventory control of production systems subject to uncertainties on demand and lead times
}

Charifa Moussaoui, Rosa Abbou, Jean-Jacques Loiseau

\section{To cite this version:}

Charifa Moussaoui, Rosa Abbou, Jean-Jacques Loiseau. Robust inventory control of production systems subject to uncertainties on demand and lead times. International Journal of Production Research, 2017, 55 (8), pp.2177-2196. hal-01701013

\section{HAL Id: hal-01701013 \\ https://hal.science/hal-01701013}

Submitted on 5 Feb 2018

HAL is a multi-disciplinary open access archive for the deposit and dissemination of scientific research documents, whether they are published or not. The documents may come from teaching and research institutions in France or abroad, or from public or private research centers.
L'archive ouverte pluridisciplinaire HAL, est destinée au dépôt et à la diffusion de documents scientifiques de niveau recherche, publiés ou non, émanant des établissements d'enseignement et de recherche français ou étrangers, des laboratoires publics ou privés. 


\title{
Robust inventory control of production systems subject to uncertainties on demand and lead times
}

\author{
Charifa Moussaoui ${ }^{\mathrm{a}}$ and Rosa Abbou $^{\mathrm{b} *}$ and Jean Jacques Loiseau ${ }^{\mathrm{b}}$ \\ ${ }^{b}$ LUNAM Université, Institut de Recherche en Communications et Cybernétique de Nantes \\ IRCCyN UMR CNRS 6397. École Centrale de Nantes, 44321 Nantes Cedex 3, France; \\ ${ }^{a}$ Laboratoire d'Informatique, Modélisation et Optimisation des Systèmes, LIMOS UMR CNRS 6158 \\ 63173 Aubière Cedex, France
}

()

\begin{abstract}
In this paper, we are interested in the controller design for constrained production systems subject to uncertainties on the demand and the production delays. The case study focuses on the inventory regulation problem in production systems which must respond to the customer demands of finite products. Such systems are characterized by the presence of delays due to production processes, the saturation of the input command and the constraints due to the finite capacities of stocks. In our study, we assume that $(i)$ the customer demands are considered to be unknown but bounded by a given value, (ii) both the control input and the inventory output are subject to assigned constraints, and (iii) the production delay is defined with an uncertainty interval. Our model includes two factors that commonly have an impact on the supply chain performances and cause the bullwhip effect: the variability of the customer demand and the uncertainty on the lead time.

The proposed approach is based on a saturated predictor-feedback structure, in which the constraints and the physical limitations of the production system are taken into account. The concepts used in this approach are the BIBO-stability and the $\mathcal{D}$-invariance properties. We examine then the bullwhip effect phenomenon, which is an important observation in supply chain management. In order to study the robustness of the control system state feedback, the proposed approach gives necessary and sufficient conditions on the controller parameters, for which the system requirements will be completely met, and permits to ensure the bounds of solutions for the control parameters. The accuracy of the proposed methodology is illustrated through simulation result which demonstrates that the bullwhip effect can be reduced, but not completely eliminated, by using a saturated command and a predictor-feedback structure.
\end{abstract}

Keywords: Inventory control, BIBO-stability, $\mathcal{D}$-invariance properties, time-delay systems, robust control, predictor-feedback structure.

\section{Introduction}

\subsection{About the inventory regulation problem}

The paper deals with the inventory regulation problem in logistical systems. A logistical system is a network of elementary devices, that interact between them, exchanging goods, money, and information. The aim of each component of the system, is to answer demands arising from each other. The demand is rapidly variable, and, since production takes time, this variation generates oscillations of the inventory levels. In (Forrester, 1973), the author analyzed the dynamics of industrial systems, and showed that unstable oscillations may happen in supply chains, which is called bullwhip effect. He recommended managing demand-driven policies that permit to coordinate the

*Corresponding author. Email: Rosa.Abbou@irccyn.ec-nantes.fr 
different actors of a supply chain, thanks to the exchange of information. Such policies may be seen as stabilizing inventory control laws as initially remarked by (Simon, 1952). (Riddalls and Bennett, 2002) proposed a control structure based on a feedback structure using a smoothing of the expected demand. They used an approximation of the delay operator by a first-order system. (Sipahi and Delice, 2008) showed that such an approximation may induce an erroneous evaluation of the stability of the system, and developed numerical methods for a precise estimation of the stability regions in the space of the control parameters.

The paper deals with the methodology of control design for constrained production systems within an uncertainty on the production delay. Because of the presence of the delay, those systems are named delayed systems, also called systems with after effect or dead-time, memory systems or hereditary systems, systems with deviating argument or differential-difference equations. They belong to the class of functional differential equations which are infinite dimensional, as opposed to ordinary differential equations (Richard, 2003). The class of delay systems include systems having a phenomenon of delay in their dynamics. Additionally, some systems would be particularly sensitive to the presence of delay, and even a small delay may drastically affect how they behave. However, although these phenomena of delays have complex effects on the properties of the system, and although they are often a source of instability, they are also known for stabilizing effects. That is why the delay in command signals is a serious issue for dynamic system that needs to act in real-time.

These features have generated a great curiosity on the part of the community of automation engineers. From the state of art, one can refer, for instance, to special issues such as (Richard, 2003), (Dion et al., 2001), (Loiseau and Rabah, 1997) and (Richard and Kolmanovskii, 1998). There are also some survey papers such as (Kharitonov, 1998), (Loiseau, 1998), (Niculescu et al., 1997), (Olbrot, 1998), (Richard, 2000), (Niculescu and Gu, 2004), (Chiasson and Loiseau, 2007), (Loiseau et al., 2009), (Krstic, 2010), (Sipahi et al., 2011). Beyond the therorical interest for the study of such models, the interest that is paid to this class of systems is justified in many applied problems. Whether applications in the fields of biology, economics, transport or telecommunications network ((Bocharov and Rihan, 2000), (Chiasson and Loiseau, 2007), (Normey-Rico and Camacho, 2007)), the time-delay models allow for more realistic modeling of the dynamics that govern processes, formalizing effects delays that are present.

\subsection{Our contribution}

In this study, the production delay is considered with an uncertainty expressed by an interval. Our objective is to study the robustness of the control law. We propose in this paper a control law based on a predictor-feedback structure as developed in (Abbou et al., 2013) and (Moussaoui et al., 2014) taking into account the uncertainty on the delay. The use of a predictor-based feedback loop is a classical idea to control time-delay systems. It was first proposed in (Manitius and Olbrot, 1979). It permits pole placement, and was shown to be a basic structure for robust control of time-delay systems (Mirkin and Raskin, 2003).

The main advantage of the controller proposed in this work is it permits the analysis and control of the variations of the instantaneous inventory level and production level, in presence of a demand that is variable (unknown) and bounded. We note that the delay and the storage and production capacities are taken into account exactly. The financial benefits of this approach can be measured by the fact to meet customer demand without incurring the penalties of breaking the stock, and also by optimizing storage costs by adopting a production strategy just in time.

Another extension, that we are interested in, was its use in disturbance attenuation. In logistic systems, the perturbation corresponds to the customers demand. The regulation aims to attenuate this perturbation effect on the inventory level. The use of a prediction has an interesting interpretation when applied to the considered systems. In that case, it comes to the consideration of the Work 
in Process (WIP) on line to determine the production level, which is widely used in production management. Indeed, in a production process, it is quite common to have the WIP which includes the unfinished items which are being fabricated but are not yet completed. Moreover, when the systems are subject to actuators saturations, the use of a saturated command is necessary to solve the control problem of those systems. Also, we analyze the robustness of the control law and give the robustness margin. For more reading, one can refers to the works of (Hu and Lin, 2001), (Kapila and Grigoriadis, 2002) and (Tarbouriech et al., 2011), to name but a few, which dealt with this topic.

In this paper, a methodology for designing a robust control for production systems with inputtime delay is developed. As results, the obtained results on the necessary and sufficient conditions on the controller parameters are summarized, under any unknown but bounded customer demand. The paper is complemented by the study of the robustness of the proposed control law due to the uncertainty of delay. The remainder of this paper is organized as follows. Section 2 describes the problem statement and the characteristics of the considered production system. In section 3 , the inventory control structure is described. The case study being devoted to the inventory control problem taking into account the saturation of the command, the methodology for the controller design is recalled and summarized in the section 4 , wherein the conditions on the controller parameters are given. In order to ensure the bounds of solutions for the control parameters, an analysis of the variation of the delay is used to study the robustness of the control system state feedback and described in the section 5 . The paper concludes with some experimental results and discussions of using the proposed approach developed in the section 6 , as well as directions for future work.

\section{Problem statement}

\section{$2.1 \quad$ Inventory control problem}

We consider an elementary production system composed of a supplying unit and a storage one. A supplying unit is characterized by a delay $\theta$ which corresponds to the time needed to accomplish the task, and by an order rate denoted $u(t)$, wich is limited by a maximum supplying order rate denoted $U_{\max }$. The storage unit is characterized by a maximum storage capacity noted $Y_{\max }$. In this study, the demands are supposed to be unknown but bounded by a certain value $d_{\max }$.

In the following, we define basic variables: the production order $u(t)$, the inventory level $y(t)$, and the consumer demand $d(t)$. Those three variables are varying throughout the time, and we consider them as real variables. The dynamic of the inventory level can be described by the following differential equation:

$$
\dot{y}(t)= \begin{cases}u(t-\theta)-d(t) & \text { for } t \geq \theta, \\ \phi(t)-d(t) & \text { for } 0 \leq t<\theta .\end{cases}
$$

The production order $u(t)$ and the consumer demand $d(t)$ are homogeneous to flows, in unit per second. The inventory level $y(t)$ is a cumulative flow, taking values in number of units. So they are functions of the time $t$ that can take exclusively non-negative values. In terms of control, $u(t)$ is the control input, $d(t)$ is an external perturbation, and $y(t)$ is the output to be controlled. One remarks that the control is delayed, which comes from the fact the production of goods or services takes time presented by $\theta$. We consider with this simple model that the delay $\theta$ is a positive constant, characteristic of the considered production system.

It is important to remark that the equation (1) is well defined for $t \geq 0$, since the control is defined from $t=0$. For $0 \leq t<\theta$, the inventory evolution depends on a function $\phi(t)$ that represents the initial work in process of the system. Its support is the interval $[0, \theta[$. To evaluate 
the inventory level function is also needed the initial value, that is $y_{0}=y(0)$.

The system (1) being given, one would like to define a management policy that permits the considered economical entity to satisfactorily answer the consumer demand. It comes down to a control problem, that consists in calculating on line the production order $u(t)$, so that the inventory level $y(t)$ be well regulated, and its oscillations due to the variations of the consumer demand $d(t)$ be well attenuated. The controller should also meet the non-negative constraints on the system variable. Mathematically, it comes down to constraints on the system variables that are interpreted as specifications of the control system, as follows.

The maximal production rate of the system is denoted $U_{\max }$, the maximal inventory capacity is denoted $Y_{\max }$, and we denote $d_{\max }$ the maximal demand rate. The controller should be designed such that, for all $t \geq 0$ :

$$
0 \leq y(t) \leq Y_{\max },
$$

with

$$
0 \leq u(t) \leq U_{\max },
$$

and for every demand function satisfying

$$
0 \leq d(t) \leq d_{\max } .
$$

\section{$2.2 \quad$ Methodology of controller design}

In automatic field, the system design or any process involves determining the characteristics of resources, such as the system sizing, as well as the development of a command to a properly functioning process. To do this, we first treat the task of developing the control, taking into account the requirements and constraints of the system, and thus address the parameterization of the controller. Once the parameters are defined a priori, with this parameterization system architecture, the design is focused on the choice of different resources or system components according to specifications, and also the controller parameterization obtained in the previous phase. This method of generic design, introduced by (Karcanias, 1994) and (Karcanias, 1995), is useful in our problematic of controller design. We showed in (Abbou et al., 2013) and in (Moussaoui et al., 2014) the interpretation of this methodology, for logistics systems. This leads to design and parameterize the various resources of the logistics system, such as choosing the rate of production or transportation, and storage capacities, depending on the needs and constraints of the system, as we shall resume in the next section.

\subsection{Management insights of the proposed approach}

In this paper, we are interested on the inventory control of production systems taking into account production delays and the specific constraints of the logistical system, under unknown demand. The proposed approach is based on the feedback predictor structure, using a saturated command, which permits the elimination of the delay impacts on the closed loop behavior. The exposed approach is applied for the inventory regulation of each production unit. A first advantage is the consideration of the saturation and the positivity constraints of the system in the methodology of controller design. The second advantage is to give necessary and sufficient conditions on the controller parameters, for which the system requirements will be completely met. The third advantage, which is sought in the study of industrial cases, is how can we consider the work in process in the decision making, in 
order to reduce - or eliminate if it's possible - the bullwhip effect on the logistical system because of the uncertainty on the customers demand.

\section{Inventory control structure}

As developed in (Moussaoui et al., 2014) and extended in (Abbou et al., 2015a) and (Abbou et al., 2015b), our proposed approach to control systems with delayed inputs is based on a predictor based feedback structure. This structure permits to stabilize the system and to compensate the delay effects present in the loop. The specifications of the production system are introduced as constraints imposed to the controller, so as to forbid any overruns on the production rates or on the inventory levels, which can cause the saturation of the production unit. The role of the controller is then to keep the production rate and so, the inventory level, as far as possible within their limits.

Linear systems with saturation functions in theirs closed loop schema are non-linear systems. Introducing a saturation function noted sat, the control law $u(t)$ takes the following form:

$$
u(t)=\underset{\left[u_{m}, u_{M}\right]}{s a t}[f(t)]= \begin{cases}u_{M} & \text { if } f(t) \geq u_{M}, \\ f(t) & \text { if } u_{m} \leq f(t) \leq u_{M} \\ u_{m} & \text { if } f(t) \leq u_{m}\end{cases}
$$

As can be seen, taking into account the saturation function involves introducing a non-linearity in the model. The non-linearity property is due to the fact that the system will switch between different operating modes, having different dynamics, depending on whether the command is saturated or not. As mentioned in (Tarbouriech et al., 2011), (Kapila and Grigoriadis, 2002) and (Hu and Lin, 2001), the effects induced by the saturation on the system stability and performances, should be carefully considered.

In this section, we are going to summarize the methodology to define the parameters of the controller law of our system as shows Figure 1, and study its effects on the closed loop behavior.

\subsection{Controller law}

Regarding to the system constraints, and the nature of the system, the control law we propose to apply is a saturated command based on a feedback predictor structure. Indeed, saturated commands are commonly used for systems with saturating actuators, and permit to take into account theirs specific limitations. It was shown to be more efficient and realistic than a linear constraint control ((Tarbouriech et al., 2011), (Hu and Lin, 2001)). On the other hand, the use of a saturated controller introduces non-linearity in the closed-loop scheme of the system, due to the sat function defined as previously. For such non-linear systems, stability conditions can be obtained by computing invariant sets in which the system trajectory remains, and in which the saturation constraints are met.

The system dynamics of the inventory be given by the relation (1) with non-zero initial conditions, the control schema of our system with a saturation command is shown on Figure 1, where the command $u(t)$ is given by:

$$
u(t)=\underset{\left[0, U_{\max }\right]}{\operatorname{sat}}\left[K\left(y_{c}-z(t)\right] .\right.
$$

The parameter $K$ is the controller gain which is used to adjust the order rates placed in each level. $y_{c}$ is the reference signal of the system, which corresponds to the reference level for the 


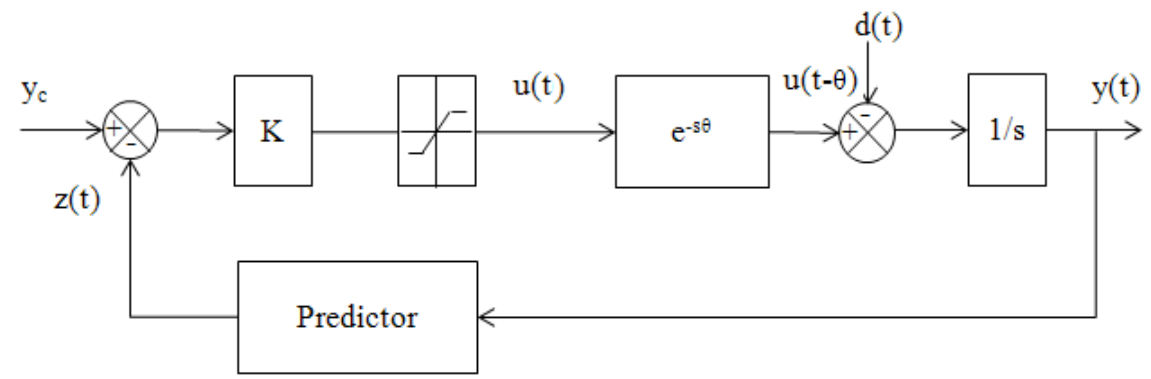

Figure 1. Closed loop schem of the system including a saturation function

inventory. $z(t)$ is the prediction of the future state of the system, that corresponds to the inventory level at $t+\theta$, as it is shown in the sequel. The feedback predictor part of the command, is used to handle the delays and the stability properties of the infinite-dimensional system, by allowing the assignment of the closed-loop system poles, in a finite number of locations in the complex plan ((Manitius and Olbrot, 1979), (Kwon and Pearson, 1980)). Also known as model reduction or Artstein reduction (Artstein, 1982), the basic idea of state prediction is to compensate the time delay $\theta$ by generating a control law that enables one to directly use the corresponding delay-free system, thanks to the prediction defined by $z(t)$ which is a prediction of the inventory level at time $(t+\theta)$, given by:

$$
z(t)= \begin{cases}y(t)+\int_{t-\theta}^{t} u(\tau) d \tau & \text { for } t \geq \theta \\ y(t)+\int_{t}^{\theta} \phi(\tau) d \tau+\int_{0}^{t} u(\tau) d \tau & \text { for } 0 \leq t<\theta\end{cases}
$$

The distributed delay $\int_{t-\theta}^{t} u(\tau) d \tau$ corresponds to the WIP during the interval $[t-\theta, t]$. The term $\int_{t}^{\theta} \phi(\tau) d \tau$ represents the initial WIP.

The prediction $z(t)$ can be rewritten, using the expression (1), as follows

$$
z(t)=y(t+\theta)+\int_{t}^{t+\theta} d(\tau) d \tau, \text { for } t \geq 0
$$

\subsection{Analysis of the closed-loop system}

We recall that the inventory and the production rate are physical resources with finite capacities. Both $y(t)$ and $u(t)$ should take exclusively non negative values, limited by the maximal capacity of the inventory $Y_{\max }$ and of the maximum production rate $U_{\max }$ respectively. Namely, $y(t)$ and $u(t)$ should be such that, for all $t \geq 0, y(t) \in\left[0, Y_{\max }\right]$ and $u(t) \in\left[0, U_{\max }\right]$, for all $d(t) \in\left[0, d_{\max }\right]$. By time derivation of the equation (1), one can see that the resulting system

$$
\dot{z}(t)=u(t)-d(t), \text { for } t \geq 0,
$$

is delay-free. The system (9) is the reduced model of the system (1)-(4). Artstein (Artstein, 1982) demonstrated that the control low $u(t)$ is admissible for the closed loop system (1)-(5) if and only if it is admissible for the system (9)-(5), and that the two systems have the same dynamics properties. Our approach is then based on the use of the reduced system (9) to design the controller such that the system constraints and requirements (2) and (3) will be fully met. 
The dynamics of the closed-loop system (9)-(5) is given by the following expression.

$$
\dot{z}(t)=\underset{\left[0, U_{\text {max }}\right]}{\text { sat }}\left[K\left(y_{c}-z(t)\right]-d(t), \text { for } t \geq 0 .\right.
$$

The stability analysis of this system is then performed by computing an invariant interval for the trajectories of system (9), in which the system constraints are met, and the BIBO stability property of the system is warranted. By studying the dynamic of $z(t)$, it is deduced that the constraint $y(t) \in\left[0, Y_{\max }\right]$ is satisfied, for every $d(t) \in\left[0, d_{\max }\right]$, if, and only if the following condition holds:

$$
z(t) \in\left[\theta d_{\max }, Y_{\max }\right] \quad \text { with } \quad \theta d_{\max } \leq Y_{\max }
$$

For more details, the reader can refer to (Moussaoui et al., 2014). In the next section, we give only the main results on controller design.

\section{Summary on controller design}

\subsection{Necessary and sufficient conditions on the controller parameters}

The controller design consists in determining suitable gain $K$ and inventory reference level $y_{c}$ for each elementary stage of the supply chain, such that the system constraints and specifications are fully met.Two main issues are to be considered. First, for given systems parameters, namely the maximum capacities $U_{\max }$ and $Y_{\max }$, the question to ask is: "is it possible to find a controller which will fully meet the constraints and the system requirements?". Then, if such a controller is indeed feasible, the second issue is about the choice of the command parameters $K$ and $y_{c}$ under the system constraints and specifications. This is the parameterization phase.

Our proposal is to determine some necessary and sufficient conditions on the controller parameters, to impose the $\mathcal{D}$-invariance property of the interval (10), so that the BIBO stability of the system and the constraints are all satisfied. These conditions are given through the following Theorem 4.1. A corollary is then formulated, which gives further results concerning the closed-loop system dynamics under Theorem 4.1 assumptions.

Theorem 4.1. Being given a system of the form (1), there exists a command of the form (5), for which the system is BIBO-stable and the constraints (2) and (3) are fulfilled, for any $d(t) \in\left[0, d_{\text {max }}\right]$ if and only if the following conditions hold true

$$
\theta d_{\max }<Y_{\max }
$$

and

$$
d_{\max } \leq U_{\max } .
$$

In addition, if the conditions (11) et (12) are met, the constraints (2) and (3) are satisfied under the control law (5) if and only if the controller parameters $\left(K, y_{c}\right)$ are such that:

$$
\theta d_{\max }+\frac{d_{\max }}{K} \leq y_{c} \leq Y_{\max }
$$

Proof. The dynamics of the closed-loop system (5)-(9) is given by the following expression.

$$
\dot{z}(t)=\underset{\left[0, U_{\text {max }}\right]}{\text { sat }}\left[K\left(y_{c}-z(t)\right]-d(t), \text { for } t \geq 0 .\right.
$$


The controller of the reduced system (14) should be designed such that the constraint (10) is fulfilled. The existence of the controller is then linked with the non-empty property of the interval ]$\left.\theta d_{\max }, Y_{\max }\right]$, which is true only when $\theta d_{\max }<Y_{\max }$. This later shows the necessity of the condition (11), its sufficiency being obvious.

The conditions (12) and (13) come from the fact that, verifying the constraint (10) at any time $t \geq 0$ implies that, the closed interval $\left[z_{\min }, z_{\max }\right]$ is $\mathcal{D}$-invariant for the system trajectories. The $\mathcal{D}$-invariance property is defined as follows.

Definition 4.1 (D-Invariance Property). Let consider a dynamic system described by $\dot{x}(t)=$ $f(x, d, t)$, with $x(0)=x_{0}$ and $d(t)$ an input of the system. A nonempty closed interval $\left[x_{1}, x_{2}\right]$ is named $\mathcal{D}$-invariant for the system described by $x(t)$ if and only if for any initial state $x(0) \in\left[x_{1}, x_{2}\right]$, and $d(t) \in \mathcal{D}, \mathcal{D}$ being a set, we have $x(t) \in\left[x_{1}, x_{2}\right]$, for all $t \geq 0$.

Formally, this property is warranted if and only if the following implications are true, for all $t \geq 0$ :

$$
x(t)=x_{1} \Rightarrow \dot{x}(t) \geq 0, \quad \text { and } \quad x(t)=x_{2} \Rightarrow \dot{x}(t) \leq 0 .
$$

Using the expression of $\dot{z}(t)$ given by (14), and provided that (4) is true, these inequalities are rewritten respectively:

$$
\underset{\left[0, U_{\max }\right]}{s a t}\left[K\left(y_{c}-z_{\min }\right)\right] \geq d_{\max },
$$

and

$$
\underset{\left[0, U_{\max }\right]}{s a t}\left[K\left(y_{c}-z_{\max }\right)\right] \leq 0 .
$$

Using the sat function definition, one can see that the inequality (15) is solvable if and only if $U_{\max } \geq d_{\max }$, that shows the sufficiency and necessity of condition (12), and thus $y_{c}$ is such that $z_{\min }+d_{\max } / K \leq y_{c}$, which, together with the condition (11) and the equality $z_{\min }=\theta d_{\max }$, establishes the sufficiency of the left part of the condition (13) of Theorem 4.1. It is necessity comes from the fact that for $y_{c}<z_{\min }+d_{\max } / K$, the inequality (15) has no solution.

The same analysis is applied for the inequality (16). This latter is solvable if and only if $y_{c} \leq z_{\max }$, which, together with equality $z_{\max }=Y_{\max }$ shows that $y_{c} \leq Y_{\max }$. This establishes the sufficiency and the necessity of the right member of the condition (13) of Theorem 4.1, and completes the proof.

The analysis of the closed-loop system dynamics shows that, under the conditions of Theorem 4.1, the system constraints and specifications are truly met. Any satisfactory controller actually permits to fill more restrictive constraints on the system variables. We describe these restrictions in the following Corollary.

Corollary 4.1. Being given a system of the form (1), with a control law of the form (5) and suitable initial conditions, such that the conditions (11), (12) and (13) are verified, then the following holds true

$$
y(t) \in\left[y_{c}-\frac{d_{\max }}{K}-\theta d_{\max }, y_{c}\right]
$$

and

$$
u(t) \in\left[0, d_{\max }\right]
$$


for all $t \geq 0$ and $d(t) \in\left[0, d_{\max }\right]$.

Proof. From expression (9), one can observe that, under Theorem 4.1 assumptions, the following implications are true for all $t \geq 0$ :

$$
z(t) \geq y_{c} \Rightarrow \dot{z}(t) \leq 0 \quad \text { and } \quad z(t) \leq y_{c}-\frac{d_{\max }}{K} \Rightarrow \dot{z}(t) \geq 0
$$

These implications show that the effective interval of variation of $z(t)$ is such that

$$
z(t) \in\left[y_{c}-\frac{d_{\max }}{K}, y_{c}\right]
$$

which represents an invariant interval for the system (9). It is seen that, under Theorem 4.1 assumptions, the interval given in (19) is included in the interval given by (10). Thus, using the expressions (5), (12) and (13), one can compute the effective interval of $y(t)$ and $u(t)$ variations which are given by the expressions (17) and (18) respectively.

The zone of admissible parameters $\left(K, y_{c}\right)$ characterized by $(13)$ is represented in Figure 2.

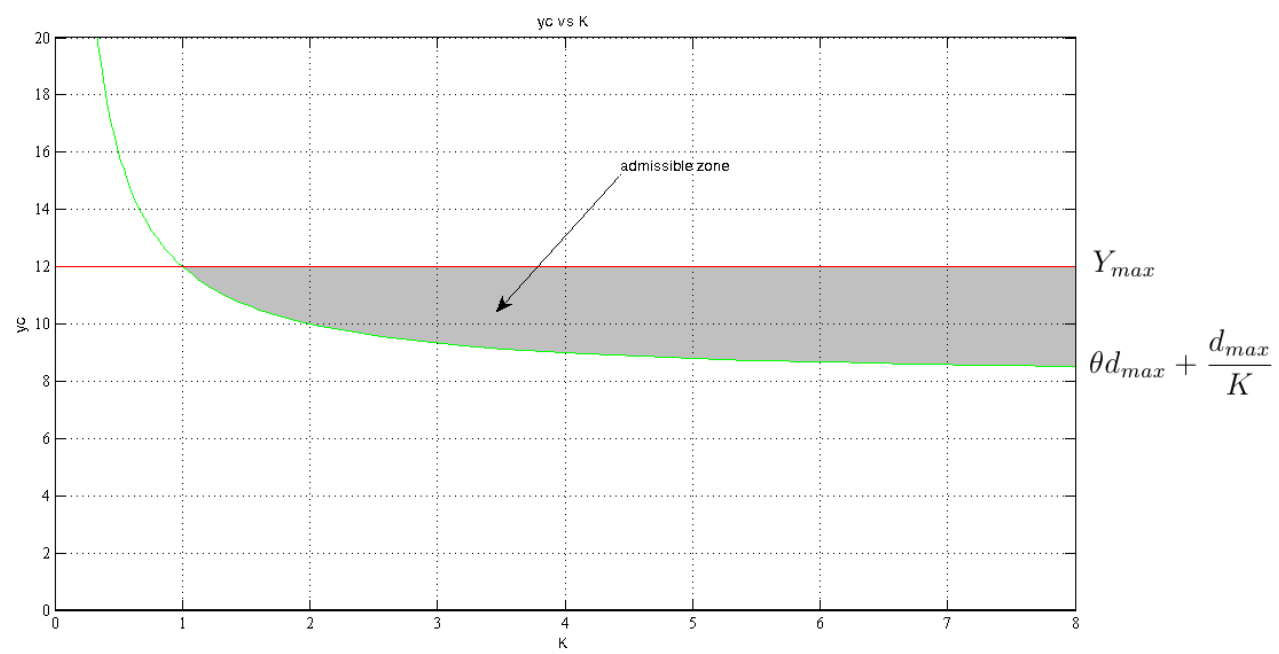

Figure 2. Zone of admissible parameters of the controller

\subsection{Admissible initial conditions}

In this study, we considered constrained systems with input-time delay as expressed by the relation (1). It follows that the inventory level evolution in the interval $[0, \theta[$, depends only on the initial conditions $\phi(t)$ and $y_{0}$, and the demand $d(t)$. In fact, the inventory level $y(t)$ is expressed by:

$$
y(t)=y_{0}+\int_{0}^{t} \phi(\tau) d \tau-\int_{0}^{t} d(\tau) d \tau, \text { for } 0 \leq t<\theta
$$

with $y_{0}$ the initial inventory at $t=0$ and $\int_{0}^{t} \phi(\tau) d \tau$ represents the intitial WIP.

We recall that $y(t)$ must also be in $\left[0, Y_{\max }\right]$ for $0 \leq t<\theta$. Considering that $\int_{0}^{t} d(\tau) d \tau \in\left[0, \theta d_{\max }\right]$, an admissible zone for the initial conditions, in which the system constraints are fulfilled, can be 
characterized by the following inequalities

$$
t . d_{\max } \leq y_{0}+\int_{0}^{t} \phi(\tau) d \tau \leq Y_{\max }, \text { for } 0 \leq t<\theta .
$$

that are readily seen to be true if and only if

$$
\theta d_{\max } \leq y_{0}+\int_{0}^{\theta} \phi(\tau) d \tau \leq Y_{\max }
$$

\section{Study of the robustness of the control law}

In this study, we consider that the real time delay of the production entity expressed by $\theta_{r}$ is not well known but defined with an uncertainty, and bounded by $\theta_{1}$ and $\theta_{2}$ such that $\theta_{r} \in\left[\theta_{1}, \theta_{2}\right], \theta_{1}$ and $\theta_{2}$ being known. We consider then an estimated delay of $\theta_{r}$, expressed by $\theta_{0}$, assumed to be known. We recall that our objective is to design a robust control law that satisfies the constraints of the system under the variation of the demand and the uncertainty on the real time delay.

\subsection{About robust control}

The robust control is a part of control theory whose approach to controller design explicitly deals with uncertainty. Robust control methods are designed to function properly provided that uncertain parameters or disturbances are found within some set. Also, robust methods aim to achieve robust performance and/or stability in the presence of bounded modeling errors. The controller is then designed to work assuming that certain variables will be unknown but bounded. In our case, the uncertainty on variables comes both from the customers demand and the production delay.

\section{$5.2 \quad$ Analysis of the closed-loop system}

The behavior of the inventory of the studied system, with non-zero initial conditions, is described by the equation:

$$
\dot{y}(t)= \begin{cases}u\left(t-\theta_{r}\right)-d(t) & \text { for } t \geq \theta_{r} \\ \phi(t)-d(t) & \text { for } 0 \leq t<\theta_{r}\end{cases}
$$

Regarding to the system constraints, the control law we propose to apply is the same as studied in (Moussaoui et al., 2014), which is a saturated command based on a feedback predictor structure. The command $u(t)$ is given by:

$$
u(t)=\underset{\left[0, U_{\max }\right]}{\operatorname{sat}}\left[K\left(y_{c}-z_{0}(t)\right)\right], \text { for } t \geq 0 .
$$

The parameter $K$ is the controller gain which is used to adjust the rate of production orders. $y_{c}$ is the reference level for the inventory. $z_{0}(t)$ is the prediction of the inventory level at time $\left(t+\theta_{0}\right)$. We can deduce that the behavior of the inventory can be expressed by:

$$
y\left(t+\theta_{r}\right)=y(t)+\int_{t-\theta_{r}}^{t} u(\tau) d \tau-\int_{t}^{t+\theta_{r}} d(\tau) d \tau
$$


As the prediction $z_{0}(t)$ is based on the estimated delay $\theta_{0}, z_{0}(t)$ is defined by :

$$
z_{0}(t)= \begin{cases}y(t)+\int_{t-\theta_{0}}^{t} u(\tau) d \tau & \text { for } t \geq \theta_{0}, \\ y(t)+\int_{t}^{\theta_{0}} \phi(\tau) d \tau+\int_{0}^{t} u(\tau) d \tau & \text { for } t<\theta_{0} .\end{cases}
$$

For $t \geq \theta_{0}$, we deduce that:

$$
z_{0}(t)=y(t)+\int_{t-\theta_{0}}^{t} u(\tau) d \tau
$$

From the relation (24), we obtain:

$$
\dot{z}_{0}(t)=u(t)-d(t)+u\left(t-\theta_{r}\right)-u\left(t-\theta_{0}\right)
$$

From the definition of $u(t)$ expressed by the relation $(21), \dot{z}_{0}(t)$ is rewritten:

$$
\dot{z}_{0}(t)=\underset{\left[0, U_{\text {max }}\right]}{\text { sat }}\left[K\left(y_{c}-z_{0}(t)\right)\right]-d(t)+\underset{\left[0, U_{\text {max }}\right]}{\operatorname{sat}}\left[K\left(y_{c}-z_{0}\left(t-\theta_{r}\right)\right)\right]-\underset{\left[0, U_{\max }\right]}{\operatorname{sat}}\left[K\left(y_{c}-z_{0}\left(t-\theta_{0}\right)\right)\right]
$$

We can remark that the relation (26) is a nonlinear and delayed equation, containing two delays, with one delay being unknown. Those delays may not be commensurable, making the study of the stability more complex, even when the system is linear, as shown in (Sipahi and Delice, 2008). To avoid this complexity, we replace the expression of $y(t)$ in the equation (22), and by definition of the prediction $z_{0}(t)$ based on the estimated time delay $\theta_{0}$, we can rewrite the dynamic of $y(t)$ as follows:

$$
y\left(t+\theta_{r}\right)=z_{0}(t)-\int_{t}^{t+\theta_{r}} d(\tau) d \tau+\int_{t-\theta_{r}}^{t-\theta_{0}} u(\tau) d \tau
$$

The term $\int_{t-\theta_{r}}^{t-\theta_{0}} u(\tau) d \tau$ corresponds to the error produced by the uncertainty on the delay $\theta_{r}$. We denote $z_{r}(t)$ the prediction of the inventory level at time $\left(t+\theta_{r}\right)$ considering the real time delay $\theta_{r}$, which is formulated by:

$$
z_{r}(t)=y(t)+\int_{t-\theta_{r}}^{t} u(\tau) d \tau
$$

From the definition of $z_{r}(t)$, we can summarize some properties.

Proposition 1. The variable $z_{r}(t)$ defined by (28) verifies the following properties:

(i) $z_{r}(t)=y\left(t+\theta_{r}\right)+\int_{t}^{t+\theta_{r}} d(\tau) d \tau$,

(ii) $\quad z_{r}(t)=z_{0}(t)+\int_{t-\theta_{r}}^{t-\theta_{0}} u(\tau) d \tau$,

(iii) $\quad \dot{z}_{r}(t)=s a t_{\left[0, U_{\max }\right]}\left(K\left(y_{c}-z_{r}(t)+\int_{t-\theta_{r}}^{t-\theta_{0}} u(\tau) d \tau\right)\right)-d(t)$.

Proof. The expression $(i)$ is equivalent to the definition of the real prediction $z_{r}(t)$.

The expression (ii) can be deduced form the relations (24) and (28). The expression (iii) is obtained by deriving the relation (28). Considering the system described by $(20)$, we obtain $\dot{z}_{r}(t)=$ $u(t)-d(t)$. Using the definition of $u(t)$, we obtain then the expression (iii). That completes the proof of the Proposition. 


\subsection{Some results on the $\mathcal{D}$-invariance properties}

In this section, we resume some results obtained on the bounds of variables, based on the $\mathcal{D}$ invariance properties.

The first $\mathcal{D}$-invariance property concerns the term $\int_{t-\theta_{r}}^{t-\theta_{0}} u(\tau) d \tau$ which is given in the following Lemma.

Lemma 1. Let consider $\alpha$ as non-negative real number. For all $\theta_{r} \in\left[\theta_{1}, \theta_{2}\right]$ and an unspecified variable $\theta_{0}$, we have:

$$
u(t) \in[0, \alpha] \Rightarrow \int_{t-\theta_{r}}^{t-\theta_{0}} u(\tau) d \tau \in\left[\min \left(0,\left(\theta_{1}-\theta_{0}\right) \alpha\right), \max \left(0,\left(\theta_{2}-\theta_{0}\right) \alpha\right)\right],
$$

In addition, these bounds are exact.

Proof. We can consider three cases:

Case 1: $\theta_{0}>\theta_{2}$ : If $\theta_{0}$ is more than $\theta_{2}$, that means $\theta_{0}$ is also more than $\theta_{1}$. Therefore, the integral $\int_{t-\theta_{r}}^{t-\theta_{0}} u(\tau) d \tau$ is positive. This term is bounded by 0 corresponding to the lower bound and by $\left(\theta_{0}-\theta_{1}\right) \alpha$, corresponding to the upper bound. in addition, those bounds are reached for, respectively, $u(t)=0$ and $u(\tau)=\alpha, \theta_{r}=\theta_{1}$.

Case 2: $\theta_{0}<\theta_{1}:$ If $\theta_{0}$ is less than $\theta_{1}$, the integral $\int_{t-\theta_{r}}^{t-\theta_{0}} u(\tau) d \tau$ is negative and evolves between $-\left(\theta_{1}-\theta_{0}\right) \alpha$ and 0 depending on the function $u$ and the value of $\theta_{r}$. The bound $\left(-\left(\theta_{1}-\theta_{0}\right) \alpha\right)$ is meet when $\theta_{r}=\theta_{2}$ and $u(\tau)=\alpha$. With the same manner, the bound 0 is meet taking $u(\tau)=0$.

Case 3: $\theta_{1} \leq \theta_{0} \leq \theta_{2}:$ The third case corresponds when $\theta_{0} \in\left[\theta_{1}, \theta_{2}\right]$. In this case, the integral $\int_{t-\theta_{r}}^{t-\theta_{0}} u(\tau) d \tau$ will take negative values if $\theta_{r}$ is in the interval $\left[\theta_{1}, \theta_{0}\right]$, and positive values if $\theta_{r}$ is in the interval $\left[\theta_{0}, \theta_{2}\right]$. Taking $u(\tau)=\alpha$ and $\theta_{r}=\theta_{1}$, the integral meets the lower bound which is $-\left(\theta_{0}-\theta_{1}\right) \alpha$, and taking $\theta_{r}=\theta_{2}$, it meets the upper bound which is $\left(\theta_{2}-\theta_{0}\right) \alpha$.

That completes the proof of the Lemma.

Based on those results obtained using the $\mathcal{D}$-invariance properties, we can generalize them through the following Lemma.

Lemma 2. Let $\alpha$ and $\beta$ be two real numbers such that $\alpha \leq \beta$. If $d_{\max } \leq U_{\max }$ and $\int_{t-\theta_{r}}^{t-\theta_{0}} u(\tau) d \tau \in[\alpha, \beta]$, then:

$$
z_{r}(t) \in\left[y_{c}-\frac{d_{\max }}{K}+\alpha, y_{c}+\beta\right], \text { for } t \geq 0 .
$$

Proof. We apply the principle of the $\mathcal{D}$-invariance. From the relation (iii) of the Proposition (1), one can remark that $\dot{z}_{r}(t) \geq 0$, for every demand $d(t) \in\left[0, d_{\max }\right]$ if the inequalities $U_{\max } \geq d_{\max }$ and $K\left(y_{c}-z_{r}(t)+\int_{t-\theta_{r}}^{t-\theta_{0}} u(\tau) d \tau\right) \geq d_{\max }$ are satisfied. That corresponds to the conditions $U_{\max } \geq d_{\max }$ and $z_{r}(t) \leq y_{c}-\frac{d_{\max }}{K}+\alpha$.

With the same manner, one can remark that $\dot{z}_{r}(t) \leq 0$ if $z_{r}(t) \geq y_{c}+\beta$. By applying the $\mathcal{D}$ invariance property, we can deduce that the interval $\left[y_{c}-\frac{d_{\max }}{K}+\alpha, y_{c}+\beta\right]$ is invariant for $z_{r}(t)$ for $t \geq 0$, under admissible initial conditions verifying this constraint. This completes the proof of the Lemma. 


\subsection{Study of robust control}

To return to our robustness study of the control law, we must ensure that there exists a control law $u(t)$ such that the output system $y(t)$ remains bounded, in order to maintain the property of BIBO-stability and the constraints on $y(t)$ are met. Based on the previous results, we can first summarize the principal results on the bounds of the variables $u(t), z_{r}(t)$ and $y(t)$ in the following Proposition.

Proposition 2. Being given a system of the form (20), with an uncertainty on the real time delay expressed by $\theta_{r} \in\left[\theta_{1}, \theta_{2}\right], \theta_{1}$ and $\theta_{2}$ being known. This system must respond to a demand verifying (4) with $d_{\max } \leq U_{\max }$. We apply on this system a control law of the form (21, 24). The following invariant sets for $u(t), z_{r}(t)$ and $y(t)$, respectively, are obtained:

(i) $u(t) \in[0, \omega]$,

(ii) $\quad z_{r}(t) \in\left[y_{c}-\frac{d_{\max }}{K}-\min \left(0,\left(\theta_{1}-\theta_{0}\right) \omega\right), y_{c}+\max \left(0,\left(\theta_{2}-\theta_{0}\right) \omega\right)\right]$,

(iii) $y(t) \in\left[y_{c}-\frac{d_{\max }}{K}-\theta_{2} d_{\max }+\min \left(0,\left(\theta_{1}-\theta_{0}\right) \omega\right), y_{c}+\max \left(0,\left(\theta_{2}-\theta_{0}\right) \omega\right)\right]$,

$$
\text { with } \omega= \begin{cases}U_{\max } & , \text { if } K \gamma \geq \frac{U_{\max }-d_{\max }}{U_{\max }}, \\ \frac{d_{\max }}{1-K \gamma} & , \text { if } K \gamma<\frac{U_{\max }-d_{\max }}{U_{\max }}\end{cases}
$$

$$
\text { and } \gamma=\max \left(\theta_{0}-\theta_{1}, \theta_{2}-\theta_{1}, \theta_{2}-\theta_{0}\right) \text {. }
$$

In addition, we verify that $d_{\max } \leq \omega \leq U_{\max }$, and $\gamma \geq \theta_{2}-\theta_{1}$.

Proof. Let be a recursive sequence $\alpha_{i}$ defined by the following recurrence:

$$
\left\{\begin{aligned}
\alpha_{0} & =U_{\max }, \\
\alpha_{i+1} & =\min \left(U_{\max }, d_{\max }+K \alpha_{i} \gamma\right), \text { for } i \geq 0
\end{aligned}\right.
$$

and $\left.\gamma=\max \left(\theta_{0}-\theta_{1}, \theta_{2}-\theta_{1}, \theta_{2}-\theta_{0}\right)\right)$. We will demonstrate by recurrence that $u(t) \in\left[0, \alpha_{i}\right]$, for $t \geq 0$ and $i \geq 0$.

First, for $i=0$, one can remark that the hypothesis $u(t)=U_{\max }$ is well verified because of the definition of the $s a t_{\left[0, U_{\max }\right]}$ function. Now, we suppose that $u(t) \in\left[0, \alpha_{i}\right]$, for $t \geq 0$. By applying Lemma 1, we deduce that $\int_{t-\theta_{r}}^{t-\theta_{0}} u(\tau) d \tau \in\left[\min \left(0,\left(\theta_{1}-\theta_{0}\right) \alpha_{i}\right), \max \left(0,\left(\theta_{2}-\theta_{0}\right) \alpha_{i}\right)\right]$. Now, by applying the Lemma (2), we obtain $z_{r}(t) \in\left[y_{c}-\frac{d_{\max }}{K}+\min \left(0,\left(\theta_{1}-\theta_{0}\right) \alpha_{i}\right), y_{c}+\max \left(0,\left(\theta_{2}-\theta_{0}\right) \alpha_{i}\right)\right]$. Considering this last interval in the the relation (ii) of the Proposition (1), we deduce that $z_{0}(t) \in\left[y_{c}-\frac{d_{\max }}{K}+\min \left(0,\left(\theta_{1}-\theta_{0}\right) \alpha_{i}\right)-\max \left(0,\left(\theta_{2}-\theta_{0}\right) \alpha_{i}\right), y_{c}+\max \left(0,\left(\theta_{2}-\theta_{0}\right) \alpha_{i}\right)-\right.$ $\left.\min \left(0,\left(\theta_{1}-\theta_{0}\right) \alpha_{i}\right)\right]$. We define $\gamma=\max \left(\theta_{0}-\theta_{1}, \theta_{2}-\theta_{1}, \theta_{2}-\theta_{0}\right)$, and we observe that $\gamma=\max \left(0,\left(\theta_{2}-\theta_{0}\right) \alpha_{i}\right)-\min \left(0,\left(\theta_{1}-\theta_{0}\right) \alpha_{i}\right)$. We deduce that $z_{0}(t)$ can be rewritten such as $z_{0}(t) \in\left[y_{c}-\frac{d_{\max }}{K}-\alpha_{i} \gamma, y_{c}+\alpha_{i} \gamma\right]$. By consequence, we deduce that $y_{c}-z(t) \in\left[-\alpha_{i} \gamma, \frac{d_{\max }}{K}+\alpha_{i} \gamma\right]$, and $u(t) \in\left[0, \min \left(U_{\max }, d_{\max }+K \alpha_{i} \gamma\right)\right]$.

From this last upper bound of $u(t)$, two cases can be considered:

- if $K \geq \frac{U_{\max }-d_{\max }}{\gamma U_{\max }}$, we verify with simplicity that $u(t)$ is bounded by an upper bound which is $U_{\max }$.

- if $K<\frac{U_{\max }-d_{\max }}{\gamma U_{\max }}$, that means $K \gamma<1$, and $(1-K \gamma) \neq 0$. We can deduce that $d_{\max } \leq$ $d_{\max }(1-K \gamma)^{-1} \leq U_{\max }$. From this relation, we verify that $u(t) \leq d_{\max }+K \gamma \alpha_{i}$, which 
permits to demonstrate the recurrence. So, in this case, $u(t)$ is bounded by $\alpha_{i}$, for every integer $i$, by consequence bounded by the limit of the recursive sequence $\alpha_{i}$, which equal to $d_{\max }(1-K \gamma)^{-1}$.

In conclusion, the recurrence $u(t) \in\left[0, \alpha_{i}\right]$, for $t \geq 0$ and for $i \geq 0$, is well verified. We deduce that $u(t) \in[0, \omega]$, for $t \geq 0$, with $\omega$ is the limit of a series $\alpha_{i}$ as the number of iterations $i$ tends to infinity. This proves the relation $(i)$ of the Proposition (2).

By applying Lemma 1 with the new bounds of $u(t)$, we obtain other new bounds of the term $\int_{t-\theta_{r}}^{t-\theta_{0}} u(\tau)$. One can deduce that $\int_{t-\theta_{r}}^{t-\theta_{0}} u(\tau) d \tau \in\left[\min \left(0,\left(\theta_{1}-\theta_{0}\right) \omega\right), \max \left(0,\left(\theta_{2}-\theta_{0}\right) \omega\right)\right]$. We obtain then the relation $(i i)$ of Proposition 2 by applying Lemma 2. The relation (iii) of Proposition 2 can be obtained with the new bounds of $z_{r}(t)$ and by applying the relation $(i)$ of Proposition 2. This completes the proof of the Proposition.

The necessary conditions to finding the parameters $K$ and $y_{c}$, depending on $\theta_{0}$, of a robust controller are expressed in the following Theorem, based on the results given in Proposition 2.

Theorem 5.1. Being given a system of the form (20), with an uncertainty on the real time delay $\theta_{r} \in\left[\theta_{1}, \theta_{2}\right], \theta_{1}$ and $\theta_{2}$ being known, there exists a command of the form (5) and (24), for which the system is BIBO-stable and the constraints (2) and (3) are fulfilled, for any $d(t) \in\left[0, d_{\text {max }}\right]$ if the following conditions hold true:

(i) $\quad d_{\max } \leq U_{\max }$

(ii) $\frac{d_{\max }}{K}+\theta_{2} d_{\max }+\omega \min \left(0, \theta_{1}-\theta_{0}\right) \leq y_{c}$,

(iii) $y_{c}+\omega \max \left(0, \theta_{2}-\theta_{0}\right) \leq Y_{\max }$.

$\omega$ being defined as described in the Proposition 2.

Proof. The conditions expressed in the Theorem 5.1 derived from the properties given in the Proposition (2). In fact, the condition $(i)$ comes from the necessary to satisfy the Proposition (2). In addition, $y(t)$ evolving in $\left[0, y_{\max }\right]$ implies that this latter contains the invariant set of $y(t)$ expressed in the relation (iii) of the Proposition 2. The condition (ii) results from the relations (ii) and (iii) of the Proposition 2. This completes the proof of this Theorem.

We can reformulate the results from the Theorem 5.1 in the following corollary, which gives further results concerning the choice of those command parameters $y_{c}, \theta_{0}$ and $K$.

Corollary 5.1. Being given a system of the form (20), with an uncertainty on the real time delay $\theta_{r} \in\left[\theta_{1}, \theta_{2}\right], \theta_{1}$ and $\theta_{2}$ being known, and under Theorem 5.1 assumptions, there exist $y_{c}$ which verifies the conditions expressed on the Theorem 5.1 if and only if the following conditions hold true:

(i) $\quad d_{\max } \leq U_{\max }$,

(ii) $\frac{d_{\max }}{K}+\theta_{2} d_{\max }+\omega \gamma \leq Y_{\max }$.

In addition, under these conditions, $y_{c}$ can be chosen in the interval:

$$
y_{c} \in\left[\frac{d_{\max }}{K}+\theta_{2} d_{\max }+\omega \min \left(0, \theta_{1}-\theta_{0}\right), Y_{\max }-\omega \max \left(0, \theta_{2}-\theta_{0}\right)\right] .
$$

Proof. The relation $(i)$ is obvious which is necessary to the Theorem 5.1. From the relations $(i i)$ and (iii) of the Theorem 5.1, one can obtain $\frac{d_{\max }}{K}+\theta_{2} d_{\max }+\omega \min \left(0, \theta_{1}-\theta_{0}\right) \leq y_{c} \leq Y_{\max }-\omega \max \left(0, \theta_{2}-\right.$ $\theta_{0}$ ). That permits to establish the relation $(i i)$ of the Corollary 5.1, taking into account the definition of $\gamma=\max \left(0, \theta_{2}-\theta_{0}\right)-\min \left(0, \theta_{1}-\theta_{0}\right)$. On the other hand, we can verify that, under the conditions 
of the Corollary 5.1, $\frac{d_{\max }}{K}+\theta_{2} d_{\max }+\min \left(0,\left(\theta_{1}-\theta_{0}\right) \omega\right) \leq Y_{\max }-\max \left(0,\left(\theta_{2}-\theta_{0}\right) \omega\right) \leq Y_{\max }$. We deduce that choosing $y_{c}$ within the range thus defined makes it possible to check the conditions (ii) and (iii) of the Theorem 5.1. This completes the proof of this Corollary.

\subsection{Design of robust controller}

In the case of the presence of an uncertainty of the delay $\theta_{r}$, the controller design consists in determining suitable gain $K$ and inventory reference level $y_{c}$, depending on the value of $\theta_{0}$, for each elementary stage of the supply chain, such that the system constraints and specifications are fully met. As explained in the section (4), the first step consists in verifying the existence of a controller which will fully meet the constraints. Then, if such a controller is indeed feasible, the second issue is about the choice of the command parameters under the system constraints and specifications. Our proposal is to determine some necessary and sufficient conditions on the controller parameters so that the BIBO-stability of the system and the constraints are all met. These conditions to choose the command parameters optimally are given through the following Theorem 5.2.

\subsubsection{Choice of command parameters $\theta_{0}, K$ and $y_{c}$}

Theorem 5.2. Being given a system of the form (20), with an estimated time delay $\theta_{0}$ and an uncertainty on the real time delay $\theta_{r} \in\left[\theta_{1}, \theta_{2}\right], \theta_{1}$ and $\theta_{2}$ being known, and under Theorem 5.1 assumptions, there exists controller parameters $\left(K, y_{c}, \theta_{0}\right)$ for a command of the form (5) and (24), for which the system is BIBO-stable and the constraints (2) and (3) are fulfilled, under admissible initial conditions, for any $d(t) \in\left[0, d_{\max }\right]$ if and only if the following condition hold true:

(i) $d_{\max } \leq U_{\max }$,

(ii) $\theta_{2} d_{\max }+\left(\theta_{2}-\theta_{1}\right) U_{\max }<Y_{\max }$.

Under these conditions, the command parameters $\theta_{0}, K$ and $y_{c}$ are determined optimally as follows:

- We choose $\theta_{0} \in\left[\theta_{1}, \theta_{2}\right]$.

- We set $K$ such as $K \geq K^{-}$, the coefficient $K^{-}$being defined by $\frac{d_{\max }}{K-}+\theta_{2} d_{\max }+\omega\left(\theta_{2}-\theta_{1}\right)=$ $Y_{\max }$, with $\omega$ as expressed in the Proposition 2.

- The parameter $y_{c}$ can be chosen in the smallest interval expressed by $y_{c} \in\left[\frac{d_{\max }}{K^{-}}+\theta_{2} d_{\max }+\right.$ $\left.\omega \min \left(0, \theta_{1}-\theta_{0}\right), Y_{\max }-\omega \max \left(0, \theta_{2}-\theta_{0}\right)\right]$.

Proof. From the relation (ii) of the Proposition 2, the control law must be designed such that the constraint on the prediction $z_{r}(t)$ must be respected. The existence of an admissible controller is linked to the existence of non-empty interval within evolves $y(t)$ as expressed in the relation (iii) of the Proposition 2, and depending on $y_{c}, \theta_{0}$ and $K$. From the definition of $\gamma=\max \left(\theta_{0}-\right.$ $\left.\theta_{1}, \theta_{2}-\theta_{1}, \theta_{2}-\theta_{0}\right)$, we remark that the minimum value of $\gamma$ of the Proposition 2 corresponds when $\theta_{0} \in\left[\theta_{1}, \theta_{2}\right]$, and therefore $\gamma=\theta_{2}-\theta_{1}$. In this case, and based on the relation (ii) from the Corollary 5.1, we deduce that $\frac{d_{\max }}{K}+\theta_{2} d_{\max }+\omega\left(\theta_{2}-\theta_{1}\right) \leq Y_{\max }$. The minimum value of $K$, named $K^{-}$, is expressed by $K^{-}=\operatorname{Inf}\left\{K \geq 0 \mid \frac{d_{\max }}{K}+\theta_{2} d_{\max }+\omega\left(\theta_{2}-\theta_{1}\right) \leq Y_{\max }\right\}$. Concerning the parameter $y_{c}$, its interval thus defined has previously been demonstrated in the Corollary 5.1. The smallest interval is obtained by replacing $K$ by $K^{-}$. We deduce that the condition $(i i)$ of the Theorem 5.2 is the necessary and sufficient condition for the existence of a solution $\left(\theta_{0}, K, y_{c}\right)$, with $d_{\max } \leq U_{\max }$. 


\subsubsection{Choice of system constraints $U_{\max }$ and $Y_{\max }$}

Under the conditions of Theorem 5.2, the analysis of the closed-loop system dynamics shows that the system constraints and specifications are truly met. Any satisfactory controller actually permits to fill more restrictive constraints on the system variables. We describe these restrictions in the following Theorem to choose the system constraints $U_{\max }$ and $Y_{\max }$ depending on $d_{\max }, \theta_{1}$ and $\theta_{2}$ which are assumed to be known and imposed.

Theorem 5.3. Being given a system of the form (20), with an uncertainty on the real time delay $\theta_{r} \in\left[\theta_{1}, \theta_{2}\right], \theta_{1}$ and $\theta_{2}$ being known, and for which the demand $d(t) \in\left[0, d_{\text {max }}\right]$ must be satisfied, with $d_{\text {max }}$ being known too. To ensure that this system could be controlled, it is necessary to choose the system constraints as follows:

$$
Y_{\max }>\left(2 \theta_{2}-\theta_{1}\right) d_{\max }, \text { and then } U_{\max } \in\left[d_{\max }, \frac{Y_{\max }-\theta_{2} d_{\max }}{\theta_{2}-\theta_{1}}[.\right.
$$

Proof. One can remark that the choice of system constraints $U_{\max }$ and $Y_{\max }$ is interdependent. In fact, from the relations $(i)$ and $(i i)$ of the Theorem 5.2, we deduce that $Y_{\max }$ must be chosen such that $Y_{\max }>\left(2 \theta_{2}-\theta_{1}\right) d_{\max }$. Furthermore, from the same relations of the Theorem 5.2, we can deduce that $U_{\max }$ must be chosen such that $U_{\max } \in\left[d_{\max }, \frac{Y_{\max }-\theta_{2} d_{\max }}{\theta_{2}-\theta_{1}}\right.$ [. This completes the proof of this Theorem.

\subsection{Admissible initial conditions}

In this study, we considered constrained systems with input-time delay as expressed by the relation (20). It follows that the inventory level evolution in the interval $\left[0, \theta_{r}[\right.$, depends only on the initial conditions $\phi(t)$ and $y_{0}$, and the demand $d(t)$. In fact, the inventory level $y(t)$ is expressed by:

$$
y(t)=y_{0}+\int_{0}^{t} \phi(\tau) d \tau-\int_{0}^{t} d(\tau) d \tau, \text { for } 0 \leq t<\theta_{r},
$$

with $y_{0}$ the initial inventory at $t=0$ and $\int_{0}^{t} \phi(\tau) d \tau$ represents the intitial WIP.

We recall that $y(t)$ must also be in $\left[0, Y_{\max }\right]$ for $0 \leq t<\theta_{r}$. Considering that $\theta_{r} \in\left[\theta_{1}, \theta_{2}\right]$, we can deduce that $\int_{0}^{t} d(\tau) d \tau \in\left[0, \theta_{2} d_{\text {max }}\right]$. An admissible zone for the initial conditions, in which the system constraints are fulfilled, can be characterized by the following inequalities

$$
t . d_{\max } \leq y_{0}+\int_{0}^{t} \phi(\tau) d \tau \leq Y_{\max }, \text { for } 0 \leq t<\theta_{r}
$$

\section{Results analysis and discussions}

In this section we illustrate the application of the different obtained results on a production unit. We use the Simulink-Matlab environment to construct a simulation model of the production system. In our case, Two cases are studied: $(i)$ the real time delay is well defined, $(i i)$ the real time delay is expressed with an uncertainty. For the two cases, we suppose that the initial conditions are satisfied and chosen such that the transitory behavior of the system is avoided.

The parameters of the studied system is as follows. The customer demand is modeled by a rectangular signal occurring between 15 and 140 time units, with $d_{\max }=240$. The maximum 
storage capacity and production are $Y_{\max }=2920$ and $U_{\max }=300$ respectively. The couple of controller parameters $\left(K, y_{c}\right)$ are determined basing on the previous theorems.

\subsection{Case 1: Time delay well estimated}

We suppose in this case that the real time delay, named $\theta$ is well known, for example $\theta=6$. We choose $K=0,6$ and in accordance with the condition (13), we choose $y_{c}=1840$. The obtained results are shown on figure (3).

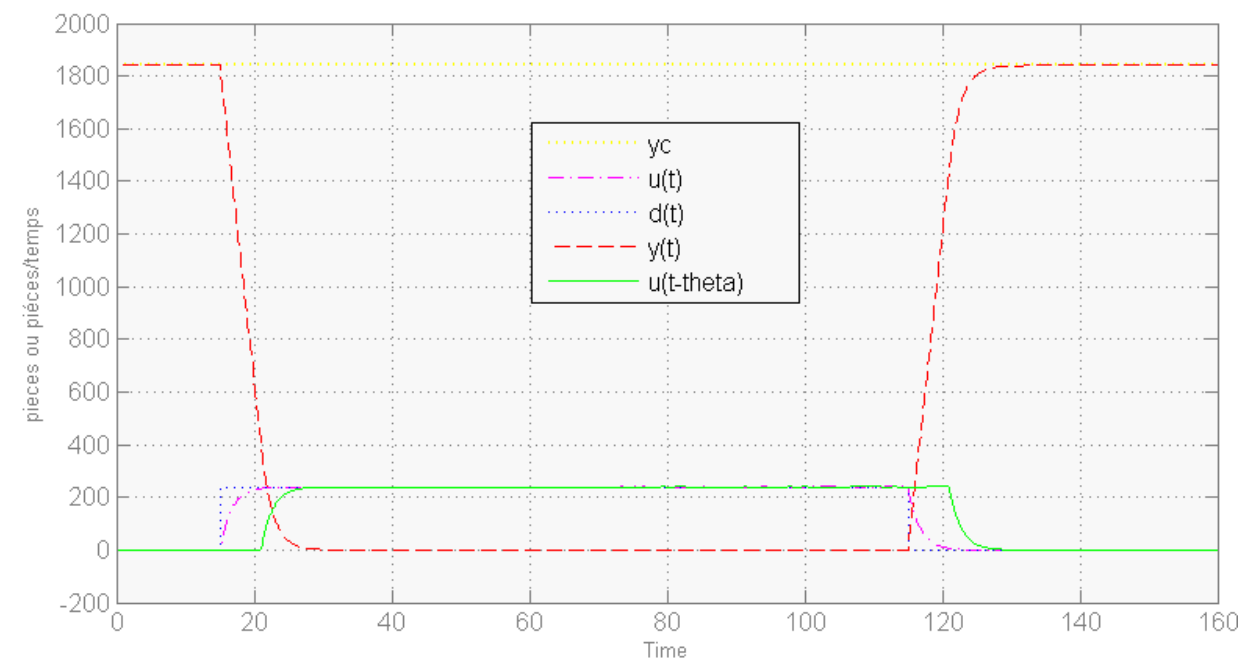

Figure 3. Results for $\theta=6, K=0,6$ and $y_{c}=1840$.

From the results, we can see that the bounds obtained in the Corollary (4.1) are met. In fact, for the fixed controller parameters, we can remark that $y(t) \in[0,1840]$ and $u(t) \in[0,240]$. We can also see that the impact of the delay is optimally compensated in the sense that the dynamic of the inventory level is fully controlled, eliminating the bullwhip effect. Furthermore, the positivity, capacity and saturation constraints are met. Another interest of the analysis when the real time delay is known is to see the impact of varying $K$ and $y_{c}$ on the system behavior, and verify the accuracy of the conditions obtained on the controllers. The impact of the demand distribution is also investigated.

\section{On the controller parameters}

From the results, one observes that the region of the acceptable controller parameters obtained for the considered control law, gives a margin for choosing those parameters. For a production system, with given values of $U_{\max }, Y_{\max }, d_{\max }$ and $\theta$, the choice of the couple $\left(K, y_{c}\right)$ belonging to the admissible zone of the parameters, is guided by the production strategy aimed. For example, choosing the set-point value for the inventory level $y_{c}$ on the lower limits of the admissible area corresponds to apply the JIT (Just In Time) strategy ${ }^{1}$. However, increasing $y_{c}$ improves the system robustness, but the inventory storage costs are also increased.

In the same way, increasing the static feedkack $K$ reduces the response time and improves system reactivity, which requires higher technology costs. Simulated results for some values of $K$ are shown on Figure 4. It can be noted among other that if the condition on the choice of the value of $K$ is not satisfied (which is the case when $K=1.5$ ), the system constraint is not respected (we obtain $y<0)$.

\footnotetext{
${ }^{1} \mathrm{JIT}$ is a production strategy that strives to improve a business return on investment by reducing in-process inventory and associated carrying costs.
} 


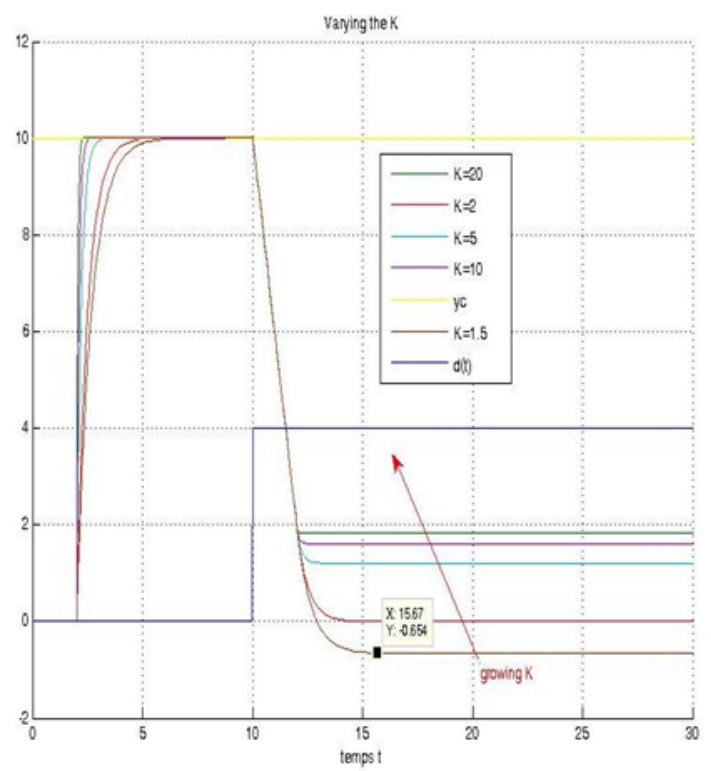

Figure 4. System outputs with variations of $K$

About the demand effects

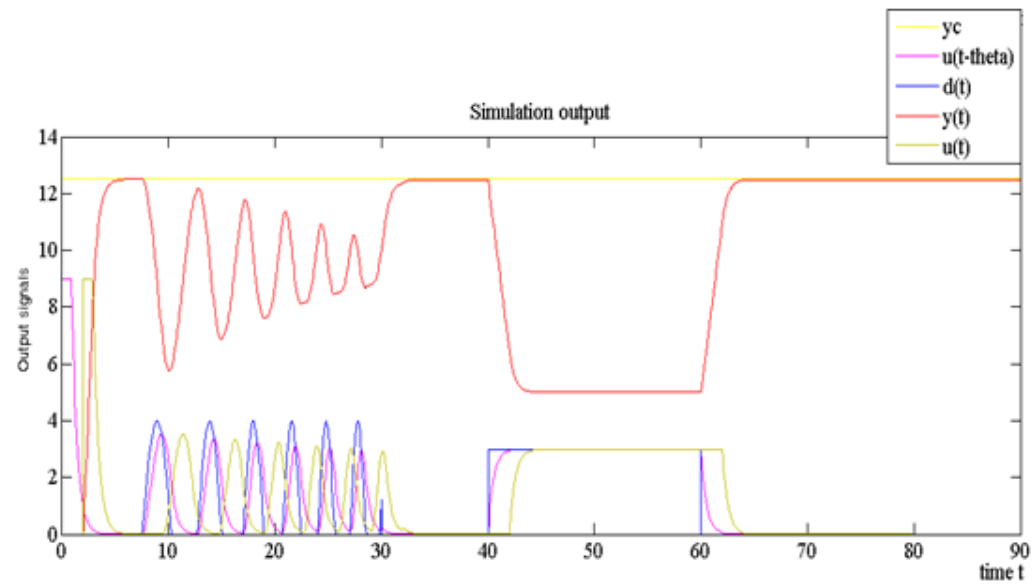

Figure 5. System outputs with variations of the demand $d(t)$

In this study, we supposed that the consumer demand $d(t)$ is unknown but bounded by the value $d_{\max }$. The system response follows closely the demand signal whether this latter is smooth or rapidly changing (Figure 5). In practical level, such tracking of the demand is not feasible: even if the production machine can bear the maximal rate of the incoming demand, the production rate cannot be changed as fast as the demand do, for technological limitation reasons. For that, the customer demand is usually forecasted and treated using a smoothing method before to be considered.

\subsection{Case 2: Time delay estimated with an uncertainty}

The second part of the simulation covers the uncertain case. The real time delay $\theta_{r}$ is expressed by an interval $\left[\theta_{1}, \theta_{2}\right]=[6,8]$, and the estimated time delay is fixed $\theta_{0}=7$. Before, we verify that 
the previous values of $Y_{\max }$ and $U_{\max }$ are admissible by the conditions on the theorem 2 . In this case, we choose the values of $K$ and $y_{c}$ according to the conditions expressed in the Theorem 5.2. We take $K=0,6$ and $y_{c}=2620$.

The results are obtained according to various values of $\theta_{r}$, the objective is to verify the obtained bounds expressed in the Theorem 5.2, and to analyze the impact on uncertainty on the real time delay on the dynamic behavior of the system, and therefore the bullwhip effect. Then, we consider 3 case studies:

- When $\theta_{r}=7$, that means the real time delay is well estimated. One can remark, from the figure $(6)$ that $y(t) \in[540,2620]$ and corresponds to the result obtained in the nominal case.

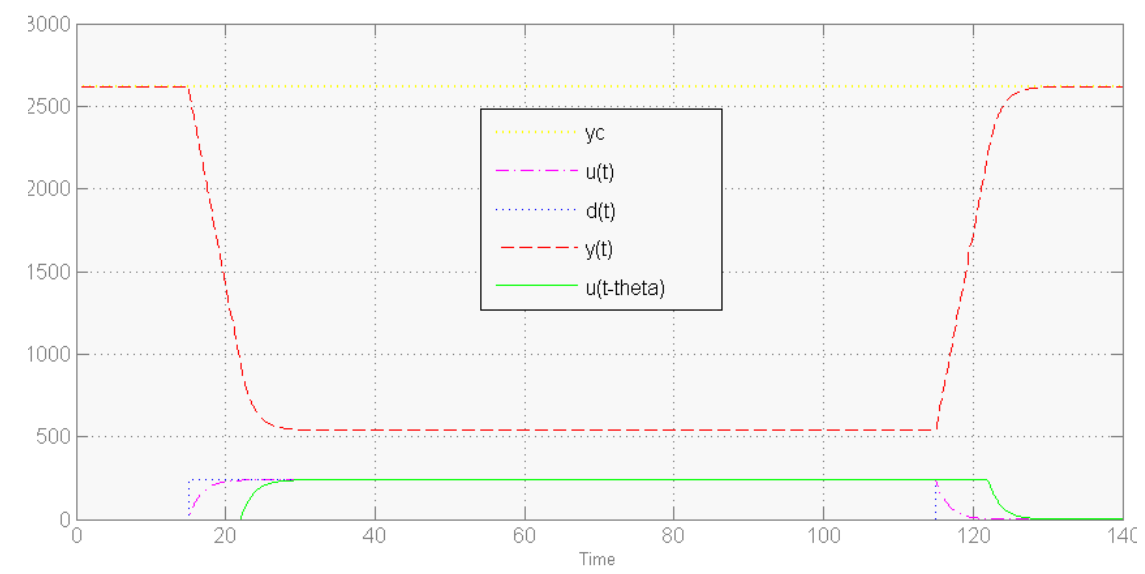

Figure 6. Results for : $y_{c}=2620, \theta_{r}=\theta_{0}=7$.

- For $\theta_{r}=6$, the result illustrated on figure (7) shows that $y(t) \in[540,2620]$. The minimum limit is reached, it corresponds to the lower bound given by the relation (iii) of the Proposition (2). We can also see short-term fluctuations on the stock level. Once the steady state is reached, these fluctuations disappear without significant overshoot.

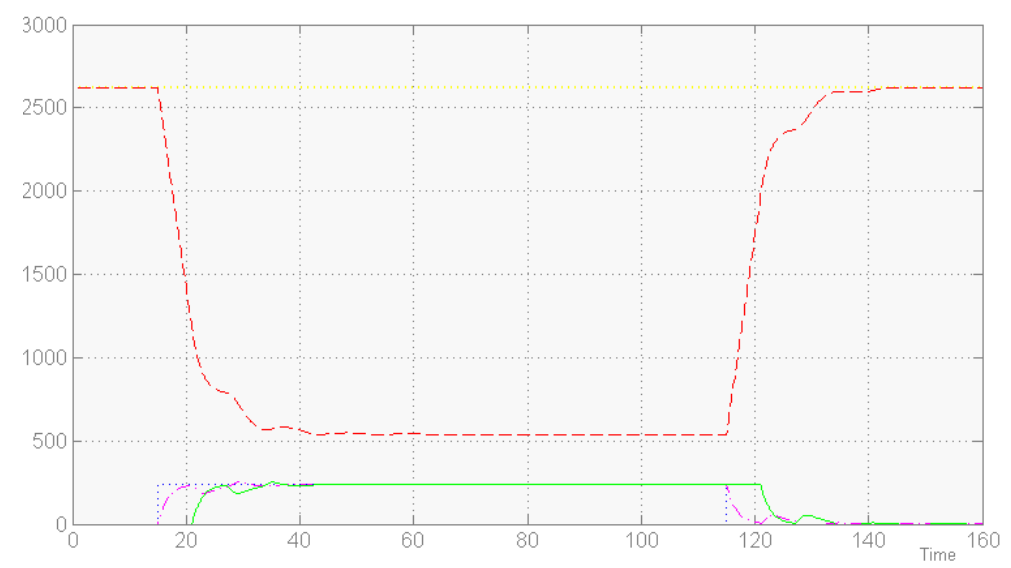

Figure 7. Results for: $y_{c}=2620, \theta_{0}=7$ and $\theta_{r}=6$.

- For $\theta_{r}=8$, we can remark that $y(t) \in[540,2860]$. In this case, it is the upper bound given in the relation (iii) of the Proposition (2), which is reached as shown in Figure (8).

We can again see the emergence of fluctuations in the stock level before stabilizing, it is important to note that the fluctuations induced on the level of production $u(t)$ are not growing 
(instead of the case of bullwhip effect, where the fluctuations are growing), the maximum amplitude of these fluctuations is an increase of almost $21 \%$ with respect to $d(t)$.

From these results, we deduce the importance to take into account the delay with maximum of precision. Indeed, in the nominal case when the delay is well estimated, the compensation of the delay by the prediction is optimal, there is no fluctuation nor overshoot observed. Such control structure helps to eliminate the bullwhip effect. In the case with uncertainty, we could test the robustness of the proposed control law. We observed the apparition of fluctuations in the stock level, as well as production orders. These fluctuations are correlated with the difference between the real delay and its estimation. It is important to notice that the control law we applied can reduce fluctuations, prevent their amplification, thus avoid the occurrence of the bullwhip effect, despite an uncertainty on the delay. On the other hand, the simulation results permit to verify the validity of the bounds obtained in the Theorems 5.2 and 5.3, and show that they are indeed met by choosing various values of the parameters of the system.

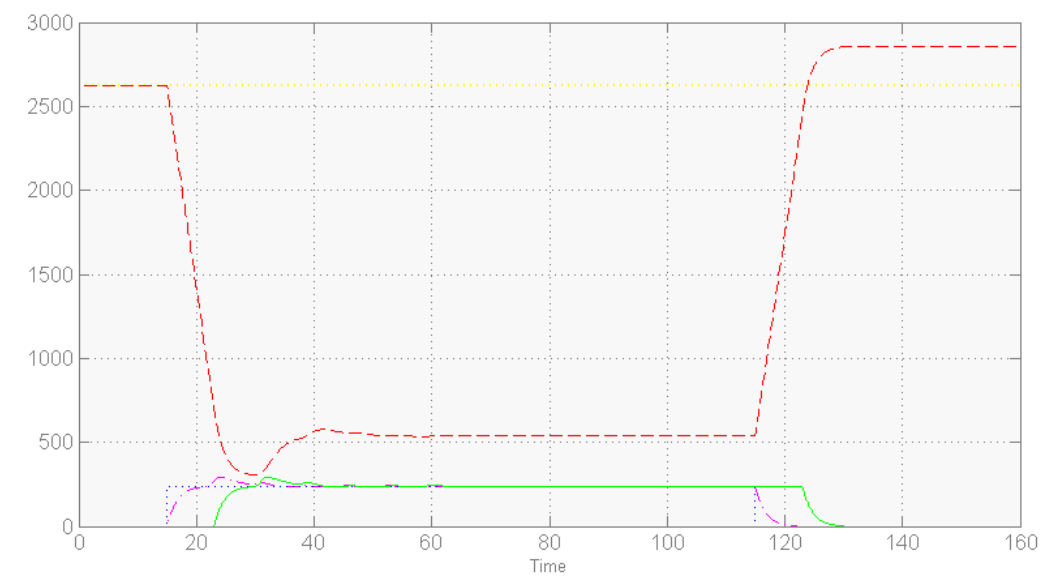

Figure 8. Results for: $y_{c}=2620, \theta_{0}=7$ and $\theta_{r}=8$.

\section{Managerial advantages of the proposed approach: Case study}

As mentioned above, a first contribution is the consideration of the saturation and the positivity constraints of the system in the methodology of controller design. The second contribution is to give necessary and sufficient conditions on the controller parameters, for which the system requirements will be completely met. The third contribution, which is sought in the study of industrial cases, is how can we consider the work in process in the decision making, in order to reduce - or eliminate if it's possible - the bullwhip effect on the logistical system because of the uncertainties on the customers demand and the production delay.

For that, let us consider a single logistic system. The main measure of the impact of the demand uncertainty on the bullwhip effect is variation ratio denoted $\lambda=\frac{d_{x}}{d_{y}}$, where $d_{x}$ corresponds to the variation of the production order, with a maximal bound denoted $\omega, d_{y}$ corresponds to the variation of the customer demand, that is bounded by $d_{\max }$. We can then define $\lambda=\frac{\omega}{d_{\max }}$. Depending on the value of $\lambda$, three cases can be considered. When $\lambda<1$, dampening of the bullwhip effect is observed, indicating a push rather than pull supply chain. If $\lambda=1$, there is no variance amplification. Otherwise, $\lambda>1$ corresponds to the presence of the bullwhip effect in the supply chain. In our example, two cases are considered: (1) the lead time is known exactly, (2) the lead time is defined with uncertainty. 


\subsection{Case of exact lead time}

When $\theta$ is well defined, we proved in (Moussaoui et al., 2014) that if the condition $d_{\max } \leq U_{\max }$, the control law $u(t)$ evolves in $\left[0, d_{\max }\right]$, so that $\omega=d_{\max }$. In this case, we obtain $\lambda=1$. That means there is no variance amplification and the bullwhip effect is eliminated. This results from the use of a control law based on predictive and saturated feedback structure.

- Illustration Example

The simulation example aims at highlighting the efficiency of the proposed inventory control to eliminate the bullwhip effect. We consider a single production system with $\theta_{0}=\theta=6, y_{c}=1840$. The figure 9 shows that, when the demand is null, the inventory level is replenished to its reference level. Also, we can see that the saturating constraint on the production order impacts the dynamics, such as the supplying process takes more time, but the demand is always fulfilled. However, the bullwhip effect is well eliminated due to the fact that there is no variance amplification.

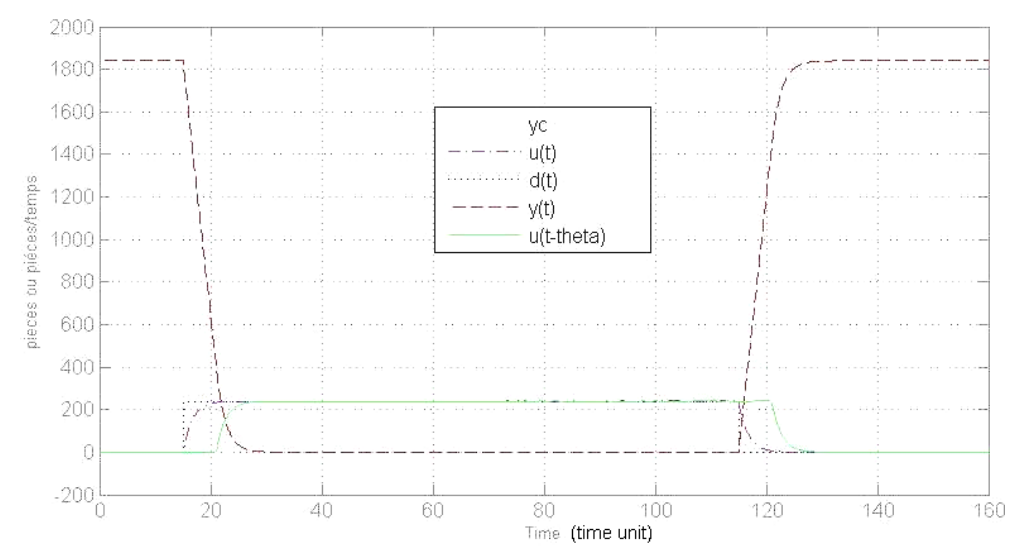

Figure 9. Impact of the demand uncertainty on the Bullwhip Effect- Case of exact lead time

\subsection{Case of lead time uncertainty}

In this case, when the lead time is defined with an uncertainty interval $\left[\theta_{1}, \theta_{2}\right], \theta_{1}<\theta_{2}$, and from the results expressed in Proposition 2, we deduce that

$$
\lambda=\frac{\omega}{d_{\max }}= \begin{cases}1 & , \text { if } K\left(\theta_{2}-\theta_{1}\right) \geq \frac{U_{\max }-d_{\max }}{U_{\max }} \\ \frac{1}{1-K\left(\theta_{2}-\theta_{1}\right)} & , \text { if } K\left(\theta_{2}-\theta_{1}\right)<\frac{U_{\max }-d_{\max }}{U_{\max }}\end{cases}
$$

If the condition $d_{\max } \leq U_{\max }$ is verified, one can remark that the impact of the lead time uncertainty on the bullwhip effect expressed by $\lambda$ can be bounded such that $1 \leq \lambda \leq \frac{U_{\max }}{d_{\max }}$. This means that although we can not completely eliminate the bullwhip effect, however, it is possible to reduce it by adjusting the value of $K$. In order to minimize the bullwhip effect, and according to the values of $U_{\max }, Y_{\max }$ and $d_{\max }$, after development of the results obtained in Theorem 5.1, we obtain the following cases.

- if $U_{\max }\left(\theta_{2}-\theta_{1}\right)+\theta_{2} d_{\max }<Y_{\max }<4 d_{\max }\left(\theta_{2}-\theta_{1}\right)+\theta_{2} d_{\max }$ then $\lambda=\frac{U_{\max }}{d_{\max }}$;

- if $4 d_{\max }\left(\theta_{2}-\theta_{1}\right)+\theta_{2} d_{\max }<Y_{\max }<\frac{U_{\max }^{2}}{U_{\max }-D_{\max }}\left(\theta_{2}-\theta_{1}\right)+\theta_{2} d_{\max }$ then $\lambda=\frac{U_{\max }}{d_{\max }}$ or $\lambda \in\left[\lambda_{1}, \lambda_{2}\right]$;

- if $4 d_{\max }\left(\theta_{2}-\theta_{1}\right)+\theta_{2} d_{\max } \leq Y_{\max }$ then $\lambda \in\left[\lambda_{1}, \frac{U_{\max }}{d_{\max }}\right]$; 
From those results, we can minimize the bullwhip effect by choosing the minimum value of $\lambda$, named $\lambda_{\min }$, such as summarized in the following Proposition.

Proposition 3. Being given a system of the form (20), and under the Theorem 5.1 assumptions, the minimum value of the variation ratio denoted $\lambda_{\text {min }}$ is calculated as follows

$$
\lambda_{\min }= \begin{cases}\frac{U_{\max }}{d_{\max }} & \text { if } Y_{1}<Y_{\max }<Y_{2} \\ \frac{1}{1-K\left(\theta_{2}-\theta_{1}\right)} & \text { if } Y_{2} \leq Y_{\max }\end{cases}
$$

with

$$
\begin{gathered}
Y_{1}=U_{\max }\left(\theta_{2}-\theta_{1}\right)+\theta_{2} d_{\max }, Y_{2}=4 d_{\max }\left(\theta_{2}-\theta_{1}\right)+\theta_{2} d_{\max } \\
K=\frac{1}{2\left(\theta_{2}-\theta_{1}\right)}\left[1-\sqrt{1-\frac{4 d_{\max }\left(\theta_{2}-\theta_{1}\right)}{Y_{\max }-\theta_{2} d_{\max }}}\right]
\end{gathered}
$$

- Illustration Example

We take the same simulation example with, in this case, $\theta_{0}=6, \theta=8, y_{c}=2620$. As we can see on the figure 10 , because of the uncertainty on the lead time, the reference level $y_{c}$ must be designed to cope with lead time uncertainty, in order to satisfy the demand without exceeding the maximum storage capacity. In addition, despite the presence of demand and delay uncertainties, the bullwhip effect is reduced.

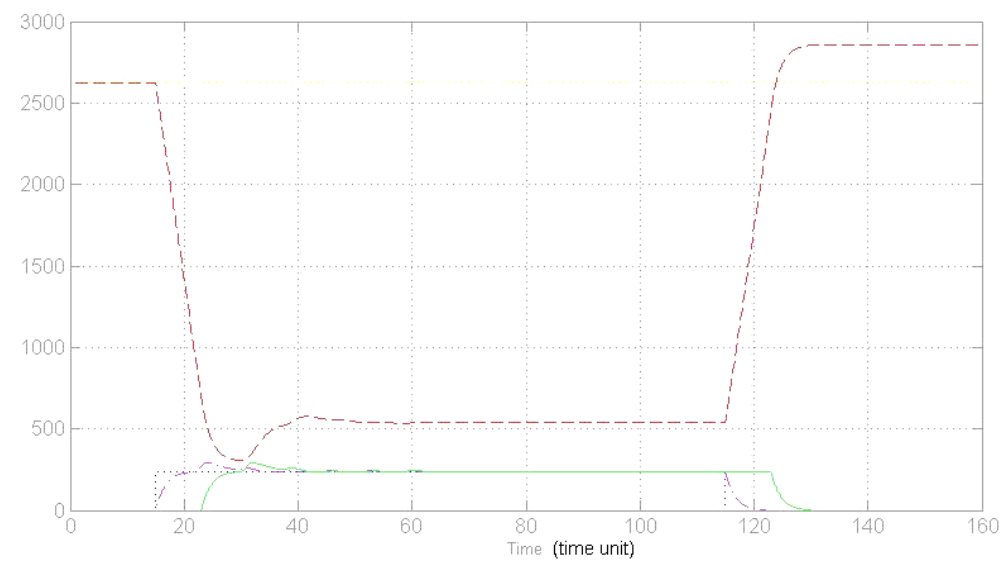

Figure 10. Impact of the demand uncertainty on the Bullwhip Effect- Case of uncertainty on the lead time

\section{Conclusion}

In this paper, we are interested in the inventory control of production systems, taking into account production delays expressed by an uncertainty and specific constraints of the production system. The proposed approach is based on the feedback predictor structure, using a saturated command, which permits the elimination of the delay impacts on the closed loop behavior. The developed approach is applied for the inventory regulation of a production unit. A first contribution is the 
consideration of the work in process in the decision making, while satisfying constraints of the system (the positivity of variables, the consideration of the maximal capacities, ...). The second contribution is to give necessary and sufficient conditions on the controller parameters, for which the system requirements will be completely met, in the presence of a pure delay $\theta$.

The third contribution consists in the study of the robustness of the control law. We considered that the real delay is not exactly well known but expressed by a uncertainty interval. We conclude that the proposed control law can reduce the fluctuations in the stock level, as well as production orders, and thus prevent their amplification and avoid the occurrence of the bullwhip effect, despite an uncertainty on the delay. Furthermore, the simulation examples show that, when the lead times are known exactly, the bullwhip effect is eliminated. However, when the lead times are defined with an uncertainty, the bullwhip effect is not eliminated but can be reduced at a minimum value. The financial benefits of this approach can be measured by the fact to meet customer demand without incurring the penalties of breaking the stock, and also by optimizing storage costs by adopting a production strategy just in time.

In our case, we considered one type of component at each node of the supply line. Further study is required to develop the proposed approach for multiple components in a node such as assembly/disassembly systems. Also, as research perspectives, it is interesting to forecast the future customers demands and integrate them in the predictive feedback structure, which should permit to ameliorate the control performance, and to have a better attenuation of the inventory oscillations. Also, it is interesting to extend the applicability of the method for the case of multiple interconnected units, by considering several actors such as suppliers, distributors, etc., and to reach the study of the Supply Chain dynamics as a whole.

\section{References}

Abbou, R., Moussaoui, C., and J. J. Loiseau. 2013. "On Controller Design for the Inventory Regulation Problem in Production Systems." 15th IFAC Symposium on Information Control Problems in Manufacturing - INCOM'15, Ottawa, May 11-13 7 (1): pp 1684-1689.

Abbou, R., Moussaoui, C., and J. J. Loiseau. 2015. "Effects of inventory control on bullwhip in logisticsystems under demand and lead time uncertainties." 15th IFAC Symposium on Information Control Problems in Manufacturing - INCOM'15, Ottawa, May 11-13 2015.

Abbou, R., Moussaoui, C., and J. J. Loiseau. 2015. "On Stability of Uncertain Time-Delay Systems: Robustness Margin for the Inventory Control." 12th IFAC Workshop on Time Delay Systems - TDS'15, Ann Arbor, Michigan, USA, 28-30 June 2015. 48 (12): pp 310-315.

Artstein, Z. 1982. "Linear systems with delayed controls: A reduction." IEEE Transactions on Automatic Control. 27 (4): pp 869-879.

Bocharov, G.A., and F.A. Rihan. 2000. "Numerical modelling in biosciences using delay differential equations." Journal of Computational and Applied Mathematics. 125 (1-2): pp 183-199.

Chiasson, J., and J. J. Loiseau. 2007. Applications of Time Delay Systems. Lecture Notes in Control and Information Sciences, Springer.

Desoer, C. A., and M. Vidyasagar. 1975. Feedback systems: Input-output properties. Academic Press.

Dion, J.M., and Dugard, L., and S.I. Niculescu. 1994. "Time delay systems." Kybernetica, Special Issue. 37 : pp 3-4.

Forrester, J.W. 1973. "Industrial dynamics." MA: MIT press, Cambridge.

Hu, T., and Lin, Z. 2001. "Control Systems with Actuator Saturation: Analysis and Design." Birkhuser.

Kapila, V., and Grigoriadis, K.M. 2002. "Actuator Saturation Control." Dekker.

Karcanias, N. 1994. "Integrated Process Design : A Generic Control Theory/Design Based Framework." Computers in Industry. 26 : pp 291-301.

Karcanias, N. 1995. "Global Process Instrumentation : Issues and Problems of a System and Control Theory Framework." Measurement. 14 : pp 103-113.

Kharitonov, V. 1998. "Robust stability analysis of time delay systems: A survey." In Fourth IFAC conference on system structure and control, Nantes, France. pp 1-12. 
Krstic, M. 2010. "Compensation of Infinite-Dimensional Actuator and Sensor Dynamics." IEEE on Control Systems. 30 (1): pp 22-41.

Kwon, M., and A. Pearson. 1980. "Feedback stabilization of linear systems with delayed control." IEEE Transactions on Automatic Control 25 (2): pp 266-269.

Loiseau, J. J., and R. Rabah. 1997. "Analysis and control of time-delay systems." JESA - European Journal of Automatic Systems, Special issue. 31 (6).

Loiseau, J. J. 1998. "Algebraic tools for the control and stabilization of time-delay systems." In First IFAC workshop on linear time delay systems, Grenoble, France. pp 234-249 .

Loiseau, J.J., Michiels, W., Niculescu, S.I., and R. Sipahi. 2009. "Topics in Time Delay Systems: Analysis, Algorithms and Control." Springer.

Manitius, A., and A.W. Olbrot. 1979. "Finite spectrum assignment problems for systems with delays." IEEE Transactions on Automatic Control 24 pp 541-553.

Mirkin, L., and N. Raskin. 2003. "Every Stabilizing Dead-Time Controller Has an Observer-Predictor-Based Structure." Automatica 39 (10) pp 1747-1754.

Moussaoui, C., Abbou, R., and J. J. Loiseau. 2014. "Controller design for a class of delayed and constrained systems: Application to supply chains." Springer. 2: pp 61-75.

Niculescu, S.I., Verriest, E.I., Dugard, L., and J.M. Dion. 1997. "Stability and robust stability of time-delay systems: A guided tour." Lecture Notes in Control and Information Sciences, Springer. Vol 228 : pp 171.

Niculescu, S.I., and K. Gu. 2004. "Advances in Time-Delay Systems." Springer Berlin Heidelberg.

Normey-Rico, J.E., and E.F. Camacho. 2007. "Control of Dead-time Processes." Springer-Verlag London Limited.

Olbrot, A.W. 1998. "Finite spectrum property and predictors." In First IFAC workshop on linear time delay systems, Grenoble, France. Plenary lecture. pp 251-260.

Richard, J.P., and V. Kolmanovskii. 1998. "Special Issue on Delay systems." Mathematics and Computers in Simulation. Vol 45: pp 3-4.

Richard, J.P. 2000. "Linear time delay systems: Some recent advances and open problems." In Second IFAC workshop on linear time delay systems. Ancona, Italy.

Richard, J.P. 2003. "Time-delay systems: an overview of some recent advances and open problems." Automatica. Vol 39: pp 1667-1694.

Riddalls, C.E., and S. Bennett. 2002. "The stability of supply chains" International Journal of Production Research. Vol 40 (2): pp 459-475.

Herbert A. Simon. 1952. "On the Application of Servomechanism Theory in the Study of Production Control" Econometrica, The Econometric Society. Vol 20 (2): pp 247-268.

Sipahi , S., and I. Delice. 2008. "Stability of Inventory Dynamics in Supply Chains with Three Delays" International Journal of Production Research. Vol 40 (2): pp 459-475.

Sipahi, R., S.-I., Niculescu, C.T., Abdallah, W., Michiels, K. and Gu. 2011. "Stability and Stabilization of Systems with Time Delay" IEEE on Control Systems. Vol 31 (1): pp 38-65.

Tarbouriech, S., G. Garcia, J.M.G. Da Silva, and I. Queinnec. 2011. "Stability and Stabilization of Linear Systems with Saturating Actuators" Springer. 